\title{
Inerties et transformations du droit de grève dans l'Espagne contemporaine
}

Josefa Dolores Ruiz Resa

\section{(2) OpenEdition}

1 Journals

\section{Édition électronique}

URL : https://journals.openedition.org/rdctss/1616

DOI : $10.4000 /$ rdctss. 1616

ISSN : 2262-9815

Éditeur

Centre de droit comparé du travail et de la sécurité sociale

\section{Édition imprimée}

Date de publication : 1 avril 2019

Pagination : 40-51

ISSN : 2117-4350

\section{Référence électronique}

Josefa Dolores Ruiz Resa, « Inerties et transformations du droit de grève dans l'Espagne

contemporaine ", Revue de droit comparé du travail et de la sécurité sociale [En ligne], 1 | 2019, mis en ligne le 01 novembre 2021, consulté le 13 novembre 2021. URL : http://journals.openedition.org/ rdctss/1616; DOI : https://doi.org/10.4000/rdctss.1616

\section{cc) (†) $\odot$}

Revue de droit comparé du travail et de la sécurité sociale est mise à disposition selon les termes de la Licence Creative Commons Attribution - Pas d'Utilisation Commerciale - Pas de Modification 4.0 International. 


\title{
INERTIES ET TRANSFORMATIONS DU DROIT DE GRÈVE DANS L'ESPAGNE CONTEMPORAINE
}

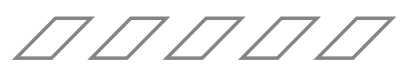

\section{RÉSUMÉ}

40 ans après son adoption, quelles transformations, la Constitution de 1978 a-t-elle produit sur la réglementation du droit de grève, après avoir été interdite et criminalisée pendant la dictature franquiste? Comment interpréter les récentes accusations criminelles formulées contre les travailleurs et syndicalistes espagnols alors qu'ils exerçaient leur droit de grève contre la réforme de la réglementation du travail de 2012? II semble qu'une sorte d'inertie antisyndicale et antigrève se manifeste à nouveau, sous l'influence d'un fort autoritarisme gouvernemental dans le domaine du travail et plus largement social. Dans cette contribution, il s'agira de voir comment certaines vieilles idées peuvent survivre dans l'Espagne de nos jours.

MOTS CLÉS: Espagne, Constitution de 1978, État de droit, droit de grève, démocratie.

\begin{abstract}
Just after 40 years of Constitution of 1978, what transformations has it produced in the regulation of the right to strike, after its prohibition and criminalization during Franco's dictatorship? How to interpret the criminal accusations of dozens of Spanish workers and trade unionists for exercising the right to strike against the situation brought by the labor reform of 2012? It seems that a kind of antiunion and anti-strike inertia is manifesting again by means of a strong governmental authoritarianism in socio-labor issues. The following pages will try to analyze to what extent these old ideas survive in present-day Spain.
\end{abstract}

KEYWORDS: Spain, Constitution of 1978, Rule of Law, Right to Strike, Democracy 


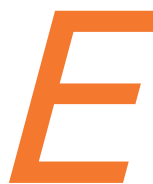

n 2014, la Confédération syndicale des Commissions ouvrières (CC.OO.) et I'Union générale des travailleurs (UGT) ont déposé une plainte auprès du Comité de la liberté syndicale de l'OIT pour atteinte au droit de grève par le Gouvernement espagnol. Les allégations portaient sur le recours croissant à certaines dispositions pénales conduisant à restreindre l'exercice du droit de grève. Était visé notamment l'article 315, paragraphe 3, du Code pénal de 19951, modifié par la suite en 2015. Cet article fait partie du Titre XV, Des délits contre les droits des travailleurs, et relève de ce qui constitue le droit pénal du travail. II prévoit des peines pouvant aller jusqu'à trois ans d'emprisonnement pour quiconque contraint d'autres personnes à commencer ou poursuivre une grève. Selon les organisations plaignantes, cet usage croissant à l'article 315, paragraphe 3 est dû à la politique répressive engagée par le gouvernement du Parti populaire contre les mobilisations sociales qui se sont multipliées suite à la réforme du marché du travail de 2012.

L'infraction visée au paragraphe 3 de l'article 315, par le Code pénal tel que modifié en 1995 et en 2015, était en fait celle visée au paragraphe 3 de l'article 496 du Code pénal de 1973 (en vigueur sous Franco), introduit par la loi 23/1976 du 19 juillet portant modification de certains articles du Code pénal. Cette loi, rédigée en pleine transition démocratique, a mis fin à la criminalisation des droits de réunion, d'association, d'expression et de liberté du travail. Mais elle a restreint pénalement l'exercice de la grève en posant la condition du respect de la liberté de travail des non-grévistes. C'est cette même liberté de travail des non-grévistes que protégerait l'article 315, paragraphe 3 du Code pénal, suite aux réformes de 1995 et de 2015.

Dans sa réponse à l'OIT, le gouvernement espagnol a fait valoir les éléments suivants: le droit de grève, tel qu'énoncé à l'article 28, paragraphe 2 de la Constitution espagnole de 1978 [ci-après CE], n'est pas un droit absolu et doit être concilié avec d'autres droits tels que la liberté de travail (article 35 CE) et la dignité de la personne (article $10 \mathrm{CE}$ ); l'article 315, paragraphe 3 du Code pénal n'a pas fait l'objet d'une application généralisée ces dernières années (avec, à l'appui, plusieurs listes de peines prononcées entre 1997 et 2009 avant la réforme du marché du travail, ainsi qu'à l'époque du gouvernement socialiste de Rodríguez Zapatero); et, la réforme du Code pénal² entreprise en 2015 par le gouvernement du Parti populaire, a allégé les peines prévues à l'article 315, paragraphe 3.

Pour sa part, le Comité de la liberté syndicale de l'OIT a recommandé au gouvernement espagnol d'évaluer l'impact de la révision de 2015 de l'article 315, paragraphe 3 du Code pénal. Mais il a noté que les peines minimales prévues pour les cas de contraintes exercées pendant la grève étaient: supérieures aux peines prévues par le Code pénal pour le délit

1 Loi organique 10/1995, du 23 novembre, du Code pénal. BOE numéro 281, du 24 novembre 1995.

2 Par la loi organique 1/2015, du 30 mars. 
général de contrainte; et, supérieures aux peines minimales prévues pour le délit général de contrainte lorsque celui-ci vise à empêcher l'exercice d'un droit fondamental ${ }^{3}$ (article 172.1). Toutefois, la liberté de travail n'est pas considérée comme un droit fondamental par la CE: cette qualité est reconnue uniquement aux Droits fondamentaux et libertés publiques, énoncés à la Section 1, Chapitre II, Titre I de la CE, parmi lesquels figure le droit de grève.

Les allégations portées devant le Comité de la liberté syndicale de I'OIT sont symptomatiques d'une certaine inertie, caractéristique du traitement de la grève par le système juridique espagnol qui a toujours considéré la grève comme une pathologie qui devrait être interdite ou, tout au plus, tolérée, puisque, apparemment, inévitable ${ }^{4}$ (des grèves ont lieu même lorsqu'elles sont interdites, comme sous le régime franquiste ${ }^{5}$ ). Si l'on ajoute à cela, la nouvelle loi adoptée en 2015, et baptisée par euphémisme, protection de la sécurité des citoyens, qui énonce de nouvelles restrictions de l'exercice du droit de grève et d'autres droits tels que celui de manifester ${ }^{6}$, l'impression de déjà-vu est encore plus frappante.

Après avoir été interdite et criminalisée sous la dictature franquiste, quel bilan peuton faire de la réglementation du droit de grève durant la période constitutionnelle la plus récente, et la plus longue, d'Espagne? Quelles sont les principales différences de réglementation induites par le passage de la grève comme délit à la grève comme droit? Comment interpréter les poursuites pénales dont ont fait l'objet ces dernières années des dizaines de travailleurs et de syndicalistes espagnols pour avoir exercé leur droit de grève face à la précarité de l'emploi et la remise en cause de leurs droits par la réforme du marché du travail de 2012?

Sur ce point, il semble que l'Espagne ne diffère pas des pays voisins: cette situation traduit l'autoritarisme croissant vers lequel bascule le capitalisme, à l'instar de la crise économique mondiale de 2008 et des changements survenus dans la perception du droit de grève sur la scène internationale ${ }^{7}$, où, là aussi, la tendance consiste plutôt à en limiter la portée. Cependant, les préjugés qui fondent les restrictions de l'exercice du droit de grève en Espagne semblent avoir leurs propres traits: une conception autoritaire du pouvoir politique, qui se manifeste par un contrôle excessif du gouvernement sur le pouvoir législatif et la production normative, à travers la promulgation de décrets-lois. Cette perception du

3 GB.328/INS/14, paragraphe 498. Sur ces affaires, J. Terradillos Basoco, "De la huelga como derecho al piquete como delito » («Du droit de grève au délit de piquet »). Au sujet de la décision sur l'affaire « Los 8 de Airbus » ("Les 8 d'Airbus »), Revista de derecho social, n 73, 2016, p. 217.

4 M. Porret Gelabert, "El derecho de huelga y sus límites », Revista técnico laboral, vol. 30, n 117, 2008, p. 3 ; M. C. Palomeque Lopez « La regulación de la huelga y sus inconvenientes funcionales ", Revista del Instituto de Estudios Económicos. Numéro monographique dédié à la réglementation du droit de grève en Espagne. n² et 3, 2010, p. 89.

5 C. Molinero Ruiz et P. Ysas, Productores disciplinados y minorías subversivas: clase obrera y conflictividad laboral en la España franquista, XXlème siècle, Madrid, 1998.

6 II s'agit de la loi organique 4/ 2015, du 30 mars. Voir à ce sujet, Orden público y restricción de las libertades. Análisis de la reforma de la legislación penal y administrativa desde la perspectiva de la protección de los derechos fundamentales. Rapport de l'association professionnelle de la magistrature d'Espagne "Jueces para la democracia », Alcalá de Henares, 20 et 21 février 2014.

7 C'est le cas, par exemple, de la position récente des employeurs, au sein de I'OIT, qui soutiennent que le droit de grève n'est reconnu dans aucune convention de l'OIT. J. Bellace, « La OIT y el derecho de huelga », Revista Internacional del Trabajo, vol. 133, 2014, n 1, p. 31. 
pouvoir tend à restreindre les voies de participation politique et l'autonomie des individus dans différents secteurs d'activités, et à minimiser, voire supplanter, les compétences normatives propres au pouvoir politique dans lequel réside la souveraineté, c'est-à-dire le pouvoir législatif.

Ces présomptions étaient profondément ancrées à l'époque de la dictature franquiste qui n'a jamais cessé d'être un État capitaliste, même si celui-ci était orchestré par le clientélisme de certains groupes fidèles à Franco. II y avait alors, une ingérence constante, au profit de ces groupes, dans la libre concurrence (un principe plus normatif qu'une loi scientifique de l'économie). Ce capitalisme clientéliste s'est accompagné d'un autoritarisme fort des gouvernements en place soumis au chef de l'État qui les nommait, à savoir, Franco. II s'est également traduit par la supériorité des sources juridiques émanant du gouvernement, comme les règlements et décrets-lois, sur les lois des Cortès. Dans ce contexte, la justice est perçue comme une nécessité qui participe de l'ordre de l'univers; la grève, au contraire, signifie désordre et conflit, et représente un fléau devant être endigué ${ }^{2}$.

Afin de répondre aux questions susmentionnées, cet article part de l'hypothèse que si la réglementation actuelle du droit de grève en Espagne a entraîné un changement qualitatif par rapport à la période précédente où la grève était interdite et criminalisée, il n'en demeure pas moins que les vestiges autoritaires du passé persistent. Parmi ces derniers figurent spécialement d'abord la mainmise de l'exécutif sur la réglementation du droit de grève. Ce contrôle excessif de l'exécutif jette le doute sur la réalité de l'État de droit dans un pays où la séparation des pouvoirs n'est pas claire. II traduit également la négation des fondements de l'État constitutionnel, puisque le non-respect de la Constitution espagnole qui exige de réglementer le droit de grève par une loi organique affaiblit son caractère normatif obligatoire. Le deuxième vestige autoritaire est la perception négative de la grève, considérée comme un trouble pathologique devant être circonscrit ou enrayé par l'intervention des autorités gouvernementales compétentes pour assurer l'ordre public.

L'objectif de cet article est de mettre en évidence ces inerties. Pour y parvenir, il s'agira d'identifier l'ensemble des éléments constitutifs du statut juridique de la grève au sein du régime de 1978 (I), et de montrer dans quelle mesure l'autoritarisme politique transparaît dans la réglementation actuelle du droit de grève et dans la remise en cause progressive de son caractère fondamental (II).

\section{I - LE STATUT JURIDIQUE DU DROIT DE GRÈVE DANS LE RÉGIME CONSTITUTIONNEL DE 1978}

Du point de vue du droit social et du droit du travail, la Constitution de 1978 marque le passage d'un système dictatorial caractérisé par un chartisme social et un dirigisme économique autoritaires, à un système dans lequel la Constitution acquiert le caractère de norme fondamentale contraignante, qui a donc force obligatoire. Dans son article premier, elle érige l'État espagnol en « un État de droit, social et démocratique ».

Il convient de souligner que le statut juridique du droit de grève est fragmenté en plusieurs dispositions normatives de rang variable, parmi lesquelles se démarquent: l'article 28, paragraphe 2 CE; le décret-loi royal [ci-après RD-L] 17/1977 du 4 mars, sur les

8 J. D. Ruiz Resa, Los derechos de los trabajadores en el franquismo (Les droits des travailleurs sous le régime franquiste), éd. Dykinson, Madrid, 2015, chapitres 1, 4 et 5. 
relations de travail, qui constitue la norme de référence pour la mise en œuvre du droit de grève en l'absence de loi organique censée la réglementer; et la décision du Tribunal constitutionnel [ci-après décision STC] 11/1981 du 8 avril, qui modifie le RD-L 17/1977 pour l'adapter à la Constitution de $1978^{9}$ en abrogeant certains de ses termes ${ }^{10}$. Toutefois, le décret-loi royal de 1977 reste une disposition normative pré-constitutionnelle. II avait été élaboré pour remplacer le précédent décret-loi de 1975 sur le règlement des conflits collectifs de travail ${ }^{11}$, et donner une réponse juridique face à l'intense mobilisation sociale et aux demandes pressantes de changement imposées à l'Espagne par les organisations internationales dans le domaine des droits de l'homme ${ }^{12}$.

Conformément à l'article 10, paragraphe $2 \mathrm{CE}$, la réglementation du droit de grève doit également être appréhendée à la lumière des conventions et traités relatifs aux droits de l'homme, ratifiés par l'Espagne. II est donc nécessaire de tenir compte de l'article 6, paragraphe 4 de la Charte sociale européenne de 1961, des articles du Pacte international relatif aux droits économiques, sociaux et culturels de 1966, des articles 13 et 14 de la Charte communautaire des droits sociaux fondamentaux des travailleurs de 1989, et des Conventions 87, 98 et 154 de I'OIT (à savoir, respectivement, la Convention sur la liberté syndicale et la protection du droit syndical de 1948, la Convention sur le droit syndical et sur la négociation collective de 1949, toutes deux ratifiées par l'Espagne en 1977, et la Convention sur la négociation collective de 1981, ratifiée par l'Espagne en 1985). Enfin, la jurisprudence du Tribunal constitutionnel, initiée par la décision STC 11/1981 précité, ainsi que la jurisprudence du Tribunal suprême ${ }^{13}$ et celle d'autres organes juridictionnels espagnols, doivent être ajoutées à cet ensemble de dispositions normatives. II faut aussi tenir compte de ce que les conventions collectives peuvent prévoir au sujet de la grève.

Ce nouveau système juridico-politique a donné lieu à une série de transformations notables concernant la grève, la plus remarquable étant le passage de la criminalisation à l'époque de la dictature franquiste, à sa consécration comme droit fondamental par l'article 28, paragraphe 2 CE. Cette disposition figure au Titre I, Chapitre II, Section 1, Des droits fondamentaux et des libertés publiques. Désormais, la grève est considérée non seulement comme une liberté de nature économique-professionnelle, mais aussi comme un droit social subjectif, fondé sur les valeurs de liberté et d'égalité ${ }^{14}$, reconnu aux " travailleurs pour la défense de leurs intérêts ». Toutefois, pour comprendre la portée de cet article de la CE de 1978 qui reconnaît le droit de grève, et les changements qu'il implique par rapport au passé récent, il est nécessaire de l'interpréter à l'aune des autres articles du texte constitutionnel : l'article 37, paragraphe 2, qui reconnaît « aux travailleurs et aux chefs d'entreprise le droit de recourir à des procédures de conflit collectif »; l'article 7 CE, qui reconnaît que les syndicats de travailleurs et les associations patronales contribuent à la

9 BOE numéro 99, du 25 avril 1981, p. 1.

10 Les termes supprimés du RD-L 17/1977 peuvent être consultés https://www.boe.es/buscar/act.php?id=BOE-A-1977-6061 (récupéré le 20/01/2019).

11 Décret-loi 5/1975, du 22 mai. BOE n 127, du 28 mai 1975, p. 11319.

12 R. Rodriguez Sanchez, «Legislación laboral y conflictos sociales: del franquismo a la democracia » in A. Reig Tapia et J. Sanchez Cervello (coords.), Transiciones en el mundo contemporáneo), URV/ UNAM, Tarragona/Mexico City, 2016, p. 581.

13 Pour une étude des décisions prononcées par ce tribunal jusqu'à la fin du XXe siècle voir $A$. Ceinos Suarez, La jurisprudencia del Tribunal Supremo en materia de huelga, éd. Comares, Grenade, 2000.

14 Fondement juridique 9 de la décision STC 11/1981 du Tribunal constitutionnel. 
" défense et à la promotion des intérêts économiques et sociaux qui leur sont propres » ; l'article 28, paragraphe $1 \mathrm{CE}$, qui reconnaît à " tous " le droit de se syndiquer librement; l'article 2, paragraphe 1 de la loi organique de 1985 sur la liberté syndicale [ci-après LOLS], qui associe l'exercice de la liberté syndicale au droit de grève, considérant que l'exercice de la première implique la mise en œuvre du second, et que la grève fait partie du contenu essentiel de la liberté syndicale (décision STC 11/1981, fondements juridiques 9 et $11)^{15}$. Cela a conduit à étendre le droit de grève aux fonctionnaires ${ }^{16}$, la liberté syndicale étant reconnue à tous (article 1, paragraphe 2 LOLS), à l'exception de ceux qui en sont expressément exclus (article 28.1 CE, en lien avec les articles 1.3 et 1.4 LOLS). II s'agit des fonctionnaires de certains services considérés comme essentiels, tels que les membres des forces armées et des corps de sécurité de l'État (à l'exception de la police locale et du personnel civil des établissements militaires) et les membres de l'organisation judiciaire et du parquet ${ }^{17}$.

L'interprétation généralement admise de ces articles montre que la CE entend permettre aux travailleurs et aux employeurs de protéger leurs intérêts et de participer à la régulation de leurs intérêts économiques et sociaux. Cela favorise la démocratie sociale également fondée sur l'article 9, paragraphe $2 \mathrm{CE}$, au titre duquel il incombe aux pouvoirs publics de faciliter la participation de tous les citoyens à la vie politique, économique, culturelle et sociale.

Toutefois, même si l'objectif de la grève consiste à défendre les intérêts collectifs des travailleurs, il faut garder à l'esprit que l'article 28, paragraphe 2 CE reconnaît le droit de grève aux travailleurs individuels et non à leurs organisations, même si l'exercice de ce droit est collectif ${ }^{18}$. Cela traduirait l'intention de ne pas utiliser une conception organique de la grève qui associe celle-ci au contrôle syndical, puisqu'elle laisse le choix au travailleur individuel d'adhérer ou non à la grève, c'est-à-dire de cesser, ou non, de travailler, de se défaire, ou non, des obligations légales prévues dans son contrat de travail ${ }^{19}$. Cependant, la grève ayant vocation à protéger les intérêts collectifs, cette cessation du travail ne

15 J. L. Monereo Perez, "La titularidad del derecho de huelga y sus manifestaciones anómalas, ilegales o abusivas. Propuestas de reforma »,-Revista del Instituto de Estudios Económicos. Numéro monographique dédié à la réglementation du droit de grève en Espagne. n² et 3, 2010, p. 192.

1617 Dans ce cas, il faudra tenir compte, entre autres, de l'article 15 du décret-loi royal 5/2015, du 30 octobre, portant approbation du texte révisé de la loi sur le statut fondamental des fonctionnaires (BOE n² 261, du 31/10/2015), auquel il faut ajouter les dispositions des Communautés autonomes ayant compétence pour légiférer sur la fonction publique dans leur domaine.

17 À cet égard, il convient également de tenir compte, entre autres, de l'article 470.2 de la loi organique 6/1985, du 1er juillet, sur le pouvoir judiciaire (BOE n 157, du 02/07/1985). Cependant, en novembre 2018, face à l'annonce d'une grève des juges, le ministère de la Justice a indiqué dans son communiqué que la part proportionnelle des jours non travaillés à la suite de la grève sera déduite de leur salaire, ce qui semble être à une reconnaissance implicite de ce droit, quoi que la loi en dise.

18 V.-A. Martinez Abascal, "La titularidad del derecho de huelga. Los sujetos titulares en el plano individual y en el plano colectivo ", Revista del Instituto de Estudios Económicos. Numéro monographique dédié à la réglementation du droit de grève en Espagne. n² et 3, 2010, p. 28.

19 À cet égard, l'article 6.4 du RD-L 11/1977 dispose que «la liberté de travail des travailleurs qui ne souhaitent pas participer à la grève doit être respectée ». 
donne pas lieu à des sanctions pénales, civiles ou professionnelles ${ }^{20}$; c'est pourquoi il est souligné qu'elle a un caractère instrumental, qu'elle « a pour fonction de protéger l'intérêt collectif ${ }^{21}$ », même si elle est de nature individuelle. Autrement dit, le droit de grève est reconnu directement au travailleur et non au travers des organisations syndicales par exemple, qui peuvent au demeurant appeler à la grève.

Deuxièmement, le caractère fondamental d'un droit implique que, conformément à l'article 53, paragraphes 1 et 2 CE, en cas de violation, la protection peut être demandée devant les tribunaux ordinaires par une action fondée sur les principes de priorité et de la procédure sommaire, et, le cas échéant, par le recours individuel d'amparo devant le Tribunal Constitutionnel (article $161 \mathrm{CE}$ ), conformément à l'article 41 de la loi organique du Tribunal constitutionnel22, si la violation est le fait des autorités publiques. Le caractère fondamental d'un droit implique également que son exercice peut être réglementé uniquement par une loi organique, conformément à l'article 82 CE. Cette loi établira les garanties nécessaires à la continuité des prestations vitales pour la communauté, tout en veillant au respect de son contenu essentiel. Cependant, déterminer le contenu essentiel d'un droit n'est pas une tâche facile ; il s'agit plutôt d'un exercice complexe devant s'inscrire dans une approche relative, temporelle, casuistique et relationnelle, telle une dialectique, puisque " aucun droit n'est absolu », comme l'indique le Tribunal constitutionnel espagnol dans le fondement juridique 9 de sa décision STC 11/1981. La résolution des conflits de droits s'effectuera au moyen d'une pondération tenant compte de la relation entre leurs contenus essentiels. À cet égard, le Tribunal constitutionnel espagnol s'inscrit dans le sillage de la doctrine allemande ${ }^{23}$.

La délimitation du contenu essentiel du droit de grève a commencé par la décision STC 11/1981 afin de déterminer si le RD-L 17/1977 était constitutionnel et applicable pour encadrer l'exercice du droit de grève, en attendant l'adoption de la loi organique prévue à cet effet ${ }^{24}$. Ainsi, la décision STC 11/1981 a rappelé que «le contenu essentiel du droit de grève consiste dans la cessation du travail, selon l'une quelconque de ses manifestations ou modalités " (fondement juridique 10), et que "la grève se caractérise par la volonté délibérée des grévistes de se mettre provisoirement hors du cadre du contrat de travail (...) afin que ses titulaires puissent temporairement se soustraire à leurs obligations juridiques contractuelles » (fondement juridique 12). Le Tribunal constitutionnel semble approuver l'idée fondamentale, contenue dans le RD-L 17/1977, que seuls les travailleurs salariés peuvent exercer le droit de grève. Les travailleurs indépendants en sont exclus dès lors qu'ils ne peuvent être provisoirement placés hors du cadre d'un contrat de travail.

20 J. L. Monereo Perez, "La huelga », in J. L. Monereo, C. Molina Navarrette, M.N. Moreno Vida, Comentario a la constitución socio-económica de España), éd. Comares, Grenade, 2002, pp. 10651068 et 1082.

21 J. L. Monereo Perez, "La huelga », op. cit., pp. 1065, 1112.

22 Loi organique 2/1979, du 3 octobre, du Tribunal constitutionnel. BOE n² 239, du 05/10/1979.

23 M. L. Balaguer Callejon, "El contenido esencial del derecho de huelga », Revista de derecho político, n³4, 1981, p. 125.

24 Le projet de loi organique de 1992 n'a pas abouti. 
D'autre part, le Tribunal constitutionnel exige, au titre du contenu essentiel du droit de grève, que la cessation du travail ait pour but de protéger les intérêts professionnels des travailleurs. Dès lors, à l'instar du RD-L 17/1977, les grèves dites politiques ne sont pas admises, à moins qu'elles ne soient liées à la défense des intérêts professionnels, comme ce fut reconnu plus tard, au sujet des grèves générales organisées contre la loi sur les pensions (1985), la réglementation en matière d'emploi et de protection contre le chômage $(1992,2002)$ ou, la réforme du gouvernement sur le travail $(1994)^{25}$.

Selon certains tenants de la doctrine juridique, le Tribunal constitutionnel aurait adopté ici une approche restrictive des intérêts des travailleurs, réduite aux intérêts professionnels et circonscrite, à son tour, au privé, par opposition au modèle de grève, plus ouvert, que reconnaît la Constitution ${ }^{26}$. Cette position aurait pour effet d'atténuer la force de la grève comme mécanisme de participation et de pression des travailleurs dans les domaines social et économique.

\section{II - LA RÉGLEMENTATION JURIDIQUE DU DROIT DE GRÈVE EN ESPAGNE, OTAGE DES INERTIES DU PASSÉ}

La réglementation du droit de grève en Espagne est révélatrice d'une dévalorisation progressive de la grève, à l'instar d'autres instruments de conflit collectif associés aux organisations syndicales, caractéristique du capitalisme mondial. La grève est reléguée au second plan, derrière la liberté d'entreprendre, fer de lance de l'économie de marchée ${ }^{27}$. La réglementation espagnole appréhende également la grève à l'image du droit social de I'Union européenne, comme un mécanisme lié à la négociation collective ${ }^{28}$ dans la sphère professionnelle et du travail. Cependant au sein du système juridique espagnol, la réglementation de la grève est aussi, et surtout, révélatrice de certaines inerties héritées du passé. Des inerties caractéristiques de la culture juridique espagnole contemporaine, dans laquelle la justice est perçue comme une nécessité qui participe de l'ordre de l'univers; la grève, au contraire, signifie désordre et conflit, devant être endigué. Elle est donc tenue à l'écart de la sphère politico-publique et confinée à celle strictement professionnelle, associée au secteur privé; le gouvernement intervient pour la réguler et la contrôler, comme si elle faisait partie de la politique intérieure et de la défense même de l'État (toutes deux dirigées par le gouvernement au titre de l'article 97 de la Constitution); le droit de grève fait ainsi l'objet d'une dévalorisation progressive en tant que droit fondamental. Examinons de plus près le mécanisme à l'œuvre.

25 M. Porret Gelabert, «El derecho de huelga y sus límites legales», op. cit., p. 8.

26 J. L. Monero Perez, «La huelga », op. cit., p. 1113.

27 J. Cruz Villalon, «Regulación y práctica del derecho de huelga en España: balance y propuestas normativas ", Revista del Instituto de Estudios Económicos. Numéro monographique dédié à la réglementation du droit de grève en Espagne. $n^{\circ} 2$ et 3,2010, p. 135, où il est fait allusion à la manière dont cette conception imprègne les institutions de l'Union européenne, comme le montre la décision de la Cour de justice des Communautés européennes du 11 décembre 2007, affaire C-438/05, où le droit de grève est soumis aux limitations découlant du droit de libre établissement des entreprises, considéré comme un principe fondamental de la Communauté.

28 A. Baylos Grau, «Replanteamientos y novedades en la regulación jurídica de la huelga », Revista de Derecho Social, n 82, 2018, p. 174. 
Tout d'abord, il convient de rappeler que, malgré la décision STC 11/1981, le contenu essentiel du droit de grève est limité par le RD-L 11/1977 du 4 mars, sur les relations de travail ${ }^{29}$, qui restreint son champ d'application au contrat de travail ${ }^{30}$. Cette limitation est à l'image du système juridique précédent et ne correspond pas à l'esprit de la Constitution de 1978. Le RD-L 17/1977 a beau élever la grève au rang de droit-liberté (et non de droit fondamental comme le fait la Constitution de 1978), il n'est pas la norme désignée pour encadrer sa mise en œuvre: le RD-L 17/1977 est une norme pré-constitutionnelle fondée sur une conception pathologique de la grève, comme en témoigne l'attribution à l'autorité gouvernementale de vastes compétences de contrôle de l'exercice du droit de grève, si caractéristique du régime précédent.

La perception de la grève à travers le prisme du RD-L 17/1977 a forgé une conception restrictive de celle-ci, limitée à la sphère économico-professionnelle. Pourtant, dans son fondement juridique 7, la décision STC 11/1981 reconnaît que «le droit de grève peut être considéré (...) comme un instrument au service de la démocratie sociale et du principe de l'égalité ». Par ailleurs, lors de l'élaboration du projet de Constitution, les amendements qui voulaient inclure dans le texte constitutionnel l'expression "professionnel », invoquant la doctrine de l'OIT et le droit comparé ${ }^{31}$, ont été rejetés. Toutefois, la conception restrictive de la grève, d'origine pré-constitutionnelle, s'impose comme l'instrument de défense des seuls intérêts professionnels; l'article 11 du RD-L 17/1977, également considéré constitutionnel, détermine quant à lui quelles sont les grèves illicites: la grève pour raisons politiques, la grève de solidarité, "sauf si elle affecte l'intérêt professionnel ", lorsqu'elle a pour objet de modifier les dispositions d'une convention collective et lorsqu'elle contrevient aux dispositions du présent décret-loi royal ou à ce qui a été convenu dans une convention collective pour la résolution de conflits.

La perception du droit de grève comme relevant davantage de la sphère « économiqueprivée " que "publique-politique », expliquerait pourquoi le Tribunal constitutionnel espagnol et les juridictions ont fini par recourir à des notions de droit privé telles que la bonne foi contractuelle ${ }^{32}$ ou la théorie de l'abus de droit, pour délimiter le contenu essentiel du droit de grève, résoudre les conflits du droit de grève avec d'autres droits; et, déterminer les circonstances d'une grève abusive ou illicite ${ }^{33}$. À cet égard, sont considérées comme des grèves abusives ou illicites, conformément à l'article 6 du RDL 17/1977, également jugé constitutionnel par la décision STC 11/1981 : «les grèves tournantes, les grèves des travailleurs situés dans des secteurs stratégiques et visant à interrompre le processus productif, les grèves du zèle et d'application du règlement et, plus généralement, toute modification collective du régime de travail autre que la grève. »Conformément à la décision

29 «L'exercice du droit de grève - tel qu'énoncé à l'article 7.1 du RD-L 1977 - s'exerce précisément par la cessation de la prestation de services par les travailleurs concernés ». Des termes similaires sont utilisés, rappelons-le, dans le cinquième fondement juridique de la décision STC 11/1981: «(...) consiste dans la cessation du travail, dans l'une quelconque de ses manifestations ou modalités ".

30 Ceci est confirmé, rappelons-le, dans le fondement juridique 12 de la décision STC 11/1981, mentionné au paragraphe précédent.

31 J. L. Monero Perez, «La titularidad del derecho de huelga y sus manifestaciones anómalas, ilegales o abusivas. Propuestas de reforma », op. cit., p. 200.

32 M. L. Balaguer Callejon, "El contenido esencial del derecho de huelga », op. cit., p. 132, attire l'attention sur le fait que la décision STC 120/1983 recours déjà à cette notion dans son fondement juridique 2.

33 C'est ce qui ressort du fondement juridique 10 du STC 11/1981. 
STC 11/1981, il existe une présomption simple, c'est-à-dire réfragable (juris tantum), selon laquelle les grèves sont abusives ou illicites ${ }^{34}$. II incombe aux travailleurs en grève d'en apporter la preuve contraire devant un tribunal. L'attribution de la charge de la preuve aux travailleurs témoigne de la suspicion de désordre qui, en Espagne, a toujours pesé sur les actions de la classe ouvrière ${ }^{35}$. Enfin, d'autres notions de droits privé sont utilisées, comme celle de «dommage » : la décision STC 11/1981 s'y réfère dans son fondement juridique 18 énonçant que le droit de grève s'arrête lorsqu'il cause à autrui « un dommage plus grave que celui que les grévistes subiraient si leurs revendications n'étaient pas entendues ». Mais la pondération entre le droit de grève et ce «dommage plus grave » causé par son exercice risque de dévaloriser le droit fondamental de grève, qui, petit à petit, se voit sacrifié au profit d'autres droits, dont le caractère fondamental n'est pourtant pas reconnu par la $\mathrm{CE}^{36}$.

Deuxièmement, il convient de noter que le décret-loi est une source juridique produite par le pouvoir exécutif, bien que son approbation ultérieure par le législateur soit requise, de sorte qu'il acquiert finalement le statut de loi (ordinaire et non, organique). Les lois organiques sont issues d'une procédure et d'un mécanisme de création différents. Le décret-loi est une disposition législative alors qu'il provient du pouvoir exécutif. II est pris pour des raisons d'extrême nécessité et d'urgence, et il doit être immédiatement traité comme un projet de loi devant les Cortès. Conformément à l'article 86, paragraphe $1 \mathrm{CE}$, les décrets-lois « ne peuvent toucher à la structure des institutions fondamentales de l'État, ni aux droits, aux devoirs et aux libertés des citoyens régis par le titre premier [où le droit de grève est reconnu], ni au régime des communautés autonomes, ni au droit électoral général. » II n'est donc pas exagéré de dire que le maintien du RD-L 17/1977 en tant que « loi » réglementant l'exercice du droit de grève constitue une violation flagrante des dispositions de la Constitution de 1978 (au moins des articles 53.1 et 86.1 précités, et 81.1 qui indique les domaines devant être réglementés par des lois organiques, notamment « ceux concernant le développement des droits fondamentaux et des libertés publiques »). Cette situation affaiblit la valeur juridique de la Constitution, en tant que norme directement contraignante, et donne lieu à une usurpation du pouvoir législatif réservé aux Cortès, conformément à l'article 66 CE, en lien avec l'article 81, paragraphe 2 CE qui réglemente la procédure d'approbation, de modification et d'abrogation des lois organiques. II s'agit d'une intervention à la fois forte et injustifiée du pouvoir exécutif dans la réglementation d'un droit social fondamental. Cette situation est caractéristique de l'ancien système juridico-politique dictatorial de l'Espagne, mais elle est contraire aux principes d'un État de droit, fondé sur la prééminence du principe de légalité, la séparation des pouvoirs et la reconnaissance du pouvoir limitatif des droits, en tant que normes juridiques de rang constitutionnel, vis-à-vis de l'action des autorités publiques.

34 Fondement juridique 10.

35 À cet égard, pourquoi ne pas inverser la présomption juris tantum, c'est-à-dire partir de la présomption simple que les grèves ne sont pas abusives, sauf preuve contraire, comme le propose J. L. Monereo, « La titularidad del derecho de huelga y sus manifestaciones anómalas, ilegales o abusivas. Propuestas de reforma », op. cit.

36 M. R. Alarcon Caracuel, « Huelga en los servicios esenciales de la comunidad», Revista del Instituto de Estudios Económicos. Numéro monographique dédié à la réglementation du droit de grève en Espagne, $n^{\circ} 2$ et 3,2010 , p. 247, le considère comme « disproportionné ». 
Quoi qu'il en soit, cette intrusion du gouvernement dans la sphère du pouvoir législatif s'est poursuivie par le maintien même du RD-L de 1977 qui donne une légitimité juridique à l'interventionnisme de "l'autorité gouvernementale » dans la réglementation ultérieure de l'exercice du droit de grève. Ainsi, l'article 10 du RD-L de 1977 confie à l'« autorité gouvernementale » la responsabilité de fixer les mesures indispensables au fonctionnement des services tenus pour essentiels: «lorsqu'une grève éclate dans des entreprises assurant des services publics de quelque nature que ce soit, ou des services de nécessité urgente et reconnue, et si les circonstances sont particulièrement graves, l'autorité gouvernementale peut prendre toutes les mesures qui lui paraissent justifiées pour assurer le fonctionnement desdits services. De même, le gouvernement pourra prendre les dispositions adéquates. » Mais cet encadrement juridique, approuvé par le Tribunal constitutionnel puisqu'il reconnaît la constitutionnalité du RD-L de 1977, va à nouveau clairement à l'encontre des dispositions de l'article 28, paragraphe 2 de la Constitution, qui prévoit sa réglementation par le pouvoir représentant le peuple espagnol, dont émane la souveraineté nationale, à savoir le pouvoir législatif (article 1.1 en lien avec l'article 66.1 de la Constitution espagnole). Ainsi, les services essentiels de la communauté, comme les services minimums de ces services essentiels sont fixés par le pouvoir exécutif (national ou de la Communauté autonome). A cet égard, on signale l'erreur habituelle des autorités gouvernementales de confondre services minimum et services essentiels ${ }^{37}$.

Troisièmement, la forte présence gouvernementale dans la réglementation de l'exercice du droit de grève renforce la prédominance de l'hétéronomie ou de l'interventionnisme gouvernemental dans le domaine des relations du travail et des droits sociaux, comme c'était le cas sous la dictature franquiste. Cet interventionnisme remet en cause et discrédite les arguments qui font valoir le risque d'une mainmise de l'État sur les travailleurs, les employeurs et leurs organisations, pour justifier l'absence de loi organique réglementant le droit de grève ${ }^{38}$.

On peut donc conclure que si la réglementation actuelle du droit de grève en Espagne bénéficie de l'encadrement juridique du RD-L 17/1977 et de la décision STC 11/81 qui le déclare constitutionnel, elle n'en est pas moins soumise à un contrôle des autorités gouvernementales aussi intense et continu qu'injustifié et contestable. Certes, l'action de cette autorité est actuellement soumise au principe de légalité, elle doit être motivée, et peut être soumise à un contrôle judiciaire; mais elle véhicule l'image de l'espace public non pas comme lieu de participation démocratique mais comme lieu où se jouent la sécurité et l'ordre publics, distinct du domaine économique dont font partie le travail et la sphère professionnelle. II n'est donc pas étonnant que la grève soit perçue, non pas comme un droit pouvant servir à articuler les intérêts des travailleurs et à les faire peser dans l'espace

37 M. C. Palomeque Lopez, "La regulación de la huelga y sus inconvenientes funcionales », op. cit., pp. 115-122. M. Porret Gelabert, «El derecho de huelga y sus límites legales », op. cit. p. 26 et 27, énumère les services que les gouvernements centraux et régionaux ont considérés essentiels jusqu'en 2008.

38 En tout état de cause, le projet contrecarré de loi organique sur la grève de 1992 a reçu le soutien des syndicats et des organisations patronales, comme le rappelle M. C. Palomeque Lopez, "La regulación de la huelga y sus inconvenientes funcionales», op. cit., p. 100. 


\section{DROIT DE GRÈVE DANS L'ESPAGNE CONTEMPORAINE}

public, mais comme une action déstabilisatrice qui doit être surveillée et endiguée. Cela expliquerait pourquoi les dispositions pénales visant à protéger la liberté de travail font l'objet d'une application plus fréquente que les dispositions prévues en cas d'atteinte à l'exercice du droit de grève ${ }^{39}$, alors même que celui-ci est reconnu par la CE comme étant un droit fondamental.

39 M. J. DOLZ LAGO, « ¿Existió alguna vez un verdadero derecho penal del trabajo? » («Y a-t-il jamais eu un véritable droit pénal du travail? »), Diario La Ley, n 9168, 2018.

\section{JOSEFA DOLORES RUIZ RESA}

Professeur au département de Philosophie du Droit, Université de Grenade.

Thèmes de recherche: Méthodologie de la connaissance et de la formation juridiques, théorie des concepts juridiques, égalité et droits humains.

\section{Publications:}

« La enseñanza del derecho en la economía del aprendizaje ", Revista Discusiones, $n^{\circ} \mathrm{XIX}$ 2017, (Numéro consacré à la Formation juridique), p. 1.

Teoría del Derecho (Théorie du droit), Tirant Lo Blanch, Valencia, 2016.

$\sim$ Los derechos de los trabajadores en el franquismo,Dykinson, Madrid, 2015. 